\title{
Reducing variability in flow cytometry
}

\section{Holden T. Maecker, J. Philip McCoy and Robert Nussenblatt}

In regard to our recent Review (Standardizing immunophenotyping for the Human Immunology Project. Nature Rev. Immunol. 12, 191-200 (2012)) $)^{1}$, we appreciate the comments by Jordi Petriz and colleagues (Subjectivity and flow cytometric variability. Nature Rev. Immunol. 25 Apr 2012 (doi:10.1038/ nri3158-c1) $)^{2}$. The Minimum Information about a Flow Cytometry Experiment (MIFlowCyt) standard ${ }^{3}$, although not a direct tool for standardization, can indeed help in the evaluation of published flow cytometry data, and it may lead authors to think more carefully about the variables in their experiments. This, in turn, should contribute to a higher quality of immunophenotyping.

With regard to Fluoresence Minus One (FMO) controls ${ }^{4}$, we would suggest that these are only necessary on a routine basis when the boundaries between positive and negative populations are not obvious from other means. When there is clear separation between the populations or when a true negative population exists within the sample or experiment, a gating control is not needed. It should also be noted that FMO controls do not account for nonspecific antibody binding, so they are imperfect gating controls when significant nonspecific antibody staining is present.

We agree with Petriz and colleagues regarding the usefulness of proficiency testing studies and the high degree of variability that can result from differential gating. They describe high inter-laboratory variability in terms of counting discrete subsets such as $\mathrm{CD} 45^{+}$or $\mathrm{CD} 34^{+}$cells; it is probable that even greater variability would be observed when counting non-discrete subsets such as $\mathrm{CD} 25^{\text {bright }}$ regulatory $\mathrm{T}$ cells or $\mathrm{CD} 38^{\text {bright }}$ plasma cells. We believe that a combination of training, common gating templates and, eventually, automated algorithms such as those being evaluated by the FlowCAP (Flow Cytometry: Critical Assessment of Population Identification Methods) project are needed to overcome this variability. We thank the authors for their insightful comments.

Holden T. Maecker is at the Institute for Immunity,

Transplantation and Infection, Stanford University

School of Medicine, Stanford, California 94305, USA.

J. Philip McCoy and Robert Nussenblatt are at the Center for Human Immunology, Autoimmunity and Inflammation, National Institutes of Health, Bethesda, Maryland 20892, USA.

J. Philip McCoy is also at the National Heart, Lung and Blood Institute, National Institutes of Health, Bethesda, Maryland 20892, USA.

Robert Nussenblatt is also at the National Eye Institute, National Institutes of Health, Bethesda, Maryland 20893, USA.

Correspondence to H.T.M. e-mail: maecker@stanford.edu doi:10.1038/nri3158-c2

1. Maecker, H. T., McCoy, J. P. \& Nussenblatt, R. Standardizing immunophenotyping for the Human Immunology Project. Nature Rev. Immunol. 12, 191-200 (2012).

2. Pachón, G., Caragol, I. \& Petriz, J. Subjectivity and flow cytometric variability. Nature Rev. Immunol. 25 Apr 2012 (doi: 10.1038/nri3158-c1).

3. Lee, J. A. et al. MIFlowCyt: the minimum information about a Flow Cytometry Experiment. Cytometry A 73 926-930 (2008).

4. Roederer, M. Spectral compensation for flow cytometry: visualization artifacts, limitations, and caveats. Cytometry 45, 194-205 (2001).

Competing interests statement

The authors declare no competing financial interests. 